\title{
A DCT-Based Driving Cycle Generation Method and Its Application for Electric Vehicles
}

\author{
Cheng Lin, Li Zhao, Xingqun Cheng, and Wenwei Wang \\ National Engineering Laboratory for Electric Vehicles, Collaborative Innovation Center of Electric Vehicles in Beijing, \\ Beijing Institute of Technology, Beijing 100081, China
}

Correspondence should be addressed to Li Zhao; zhaoli@bit.edu.cn

Received 2 November 2014; Revised 7 May 2015; Accepted 20 May 2015

Academic Editor: Oleg V. Gendelman

Copyright (C) 2015 Cheng Lin et al. This is an open access article distributed under the Creative Commons Attribution License, which permits unrestricted use, distribution, and reproduction in any medium, provided the original work is properly cited.

\begin{abstract}
Nowadays, many widely used driving cycle (DC) representing and generating methods are designed for traditional vehicles with internal combustion engines (ICE). The real-world driving is viewed as a sequence of acceleration, cruise, deceleration, and idle modes. The emission and fuel consumption in each period should be taken into account carefully. However, for electric vehicles (EVs), most of them are powered by low or zero-emission renewable energy sources. The working status and energy management algorithms of them are very different from traditional vehicles. To facilitate the EV design, we proposed a novel DC representing and construction method to generate DCs for EVs. The whole driving route is divided into several length-fixed segments and each of these segments is converted into a frequency sequence. After doing that, we can adjust the frequency and amplitude of the generated driving cycle directly. The experiment results showed that the proposed method was effective and convenient.
\end{abstract}

\section{Introduction}

Recently, the rising cost of gasoline and the increasingly stringent fuel-efficiency standards make electric vehicles a worthwhile alternative to vehicles with internal combustion engine [1-5]. As an important research tool, driving cycle is widely used in the areas of traffic management, vehicular pollution measurement, and fuel consumption estimation. In general, DC gives a speed-time profile of driving behavior in a specific area or city [6-8]. For example, the US Federal Test Procedure (FTP), the Urban Dynamometer Driving Schedule for Heavy-Duty Vehicles (UDDSHDV), and the New European Driving Cycle (NEDC) were developed for different countries or cities. In most of these DCs, the realworld driving is viewed as a sequence of acceleration, cruise, deceleration, and idle modes. This is suitable for designing an internal combustion (IC) power train, in which the emission and fuel consumption in each period should be taken into account carefully. However, the electric vehicle or some of hybrid EVs are powered by low or zero-emission renewable energy sources and the energy consumed in the idle period is very low or close to zero. Additionally, most of the electric vehicles have an energy-recovery system and the running efficiencies of them are largely decided by matching the electromotor, batteries, driving cycles, and so on. The traditional DC generation and representing methods are no longer suitable for EVs or HEVs to predict the real working condition and to indicate the residual energy or travel distance. An adapted and representative driving cycle and a new DC constructing method are needed to facilitate the design of them.

However, without a special DCs generation method for electric vehicles, a large number of studies on EV design and energy management algorithm have to use the traditional driving cycles as their objective functions. Won and Langari presented a fuzzy logic based control system and evaluated it via computational simulation under the FTP75 (Federal Test Procedure) urban drive cycle [9]. Lin et al. used dynamic programming (DP) to find the optimal control actions and defined a cost function over the Urban Dynamometer Driving Schedule for Heavy-Duty Vehicles DC [10]. Rodatz et al. proposed an original concept to convert the electrical power flow into equivalent hydrogen cost [11]. And then, they tested the proposed consumption minimization strategy on the New European Driving Cycle (NEDC). Delprat et al. developed a global optimization algorithm to derive a 
real-time control strategy and tested the algorithm on the NEDC and a highway DC [12]. Kim and Peng developed a combined power management and design optimization framework and tested it for three different DCs [13]. Assanis et al. described a methodology for integrating vehicle and engine simulations and tested it for the SAE J1711 DC [14]. Gao and Porandla used simulated annealing and genetic algorithm to optimize the design of a parallel hybrid electric vehicle [15]. A combined FTP75/Highway Fuel Economy Test (HWFET) DC was used to optimize a parallel hybrid electric power train design. Zhang et al. proposed an optimization method which has the capability of providing a set of tradeoff optimal solutions among the fuel economy and various emissions and tested it with a combined UDDS/HWFET DC [16]. Liu et al. developed a real-coded, adaptive based hybrid genetic algorithm and applied it to the optimal sizing of a series hybrid electric vehicle [17]. The performance of the proposed algorithm was verified with the City-Hwy test procedure in the ADVISOR environment.

Additionally, a system design or controller configuration may be best for a given cycle but not robust for another cycle. The parameters optimized for a special DC are not necessarily optimal for others or for the entire driving profile [18-20]. Sciarretta and Guzzella believed that the control and design parameters that perform well under a given conditions may lead to poor behavior under different conditions [21]. Fugel et al. showed that the fuel consumption obtained on standard DCs does not sufficiently correlate with the consumption obtained in real conditions [22]. For electric buses and special purpose vehicles (such as electric sanitation vehicle, electric garbage truck, and electric tank car), the working and running routes are determined and the traditional driving cycles can not be used for the special driving mission profiles. An adapted and representative driving cycle has to be constructed to facilitate the design of them.

So, in this paper, we introduce a new definition of driving cycle (distance-based driving cycle, DBDC) and present a novel methodology to generate it. With the proposed methodology, a representative DBDC can be easily produced and the stochastic parameters can be determined by observed data or be manually adjusted. The generated DBDC represents the stochastic nature of the observed data. The proposed methodology gives the following advantages that are important for EVs or HEVs optimization.

(1) A novel representing method for driving cycleDBDC-was proposed, with which a driving cycle can be presented by a frequency sequence. The components which appeared in this sequence are orthogonal and can be adjusted independently. It is more advantageous than many traditional representing methods, in which many parameters are dependent on each other.

(2) A new DC generation method was proposed, with which the original DBDC array can be compressed into several frequency coefficients and these coefficients can be determined from observed data or be manually adjusted. The generated driving profiles can represent the stochastic nature of the observed data.
(3) The proposed method is very useful for electric vehicle design, and the independent frequency coefficients are very useful for testing the compatibility of electromotor, batteries, power train, and so on.

The rest of this paper is organized as follows. We first review the related work on the discrete cosine transform (DCT). Secondly, we represent the DCT-based approach for constructing distance-based drive cycle. Then we describe several experiments in which different DBDC-construction methods were compared. And with these experiments, we demonstrate how to use the methodology to build effective and efficient DBDCs. Finally, we conclude this paper by highlighting the key contributions of this work.

\section{Related Work}

2.1. Discrete Cosine Transform (DCT). A discrete cosine transform is a finite sequence of data points in terms of a sum of cosine functions oscillating at different frequencies. It was first defined by Ahmed et al. in 1974 and widely applied in science and engineering, especially in lossy compression of audios (e.g., MP3) and images (e.g., JPEG) [23]. It is very similar to the discrete Fourier transform (DFT), but using only real numbers. There are 8 standard DCT variants and only 4 of them are commonly used. The main feature of it is the property of energy compaction: most of the information in a given signal is concentrated in a few low-frequency components.

Formally, the discrete cosine transform is a linear, invertible function $f: R^{N} \rightarrow R^{N}$ (where $R$ denotes the set of real numbers) or equivalently an invertible $N \times N$ square matrix. There are several variants of the DCT with slightly modified definitions and the four commonly used forms are DCTI, DCT-II, DCT-III, and DCT-IV. In each of them, $N$ real numbers $x_{0}, \ldots, x_{N-1}$ are transformed into $N$ real numbers $X_{0}, \ldots, X_{N-1}$ according to the formulas below:

\section{DCT-I:}

$$
\begin{aligned}
X_{k}=\frac{1}{2}\left(x_{0}+(-1)^{k} x_{N-1}\right)+\sum_{n=1}^{N-2} x_{n} \cos \left[\frac{\pi}{N-1} n k\right] \\
k=0,1, \ldots, N-1 .
\end{aligned}
$$

DCT-II:

$$
X_{k}=\sum_{n=0}^{N-1} x_{n} \cos \left[\frac{\pi}{N}\left(n+\frac{1}{2}\right) k\right] \quad k=0,1, \ldots, N-1 .
$$

\section{DCT-III:}

$$
\begin{aligned}
X_{k}=\frac{1}{2} x_{0}+\sum_{n=1}^{N-1} x_{n} \cos \left[\frac{\pi}{N} n\left(k+\frac{1}{2}\right)\right] & \\
k & =0,1, \ldots, N-1 .
\end{aligned}
$$




$$
\begin{aligned}
X_{k}=\sum_{n=0}^{N-1} x_{n} \cos \left[\frac{\pi}{N}\left(n+\frac{1}{2}\right)\left(k+\frac{1}{2}\right)\right] \\
\quad k=0,1, \ldots, N-1 .
\end{aligned}
$$

2.2. Distance-Based Drive Cycle (DBDC). Driving cycle is the set of data points representing vehicle speed versus time, which can provide a wide range of applications and the most important usages of it are vehicle fuel consumption estimate and pollutants estimate $[24,25]$. Many driving cycles have been developed and most of them are designed for vehicles with traditional internal combustion engines, instead of electric vehicles (EVs) and hybrid EVs (HEVs) [26-29]. For an internal combustion engine, the energy consumed in stop-and-go scenario is very vast. However, for EV, no electric energy will be consumed in the idle period. The research emphasis of EV or HEV is not the fuel consumption or pollution, but the electric energy saving and the performance of the given vehicle. The drawback of the traditional DC generating methods is that they pay too much attention to characteristics in time domain. However, the frequency characteristics of DCs have the same importance on energy saving, vehicle performance, pollution, and so on. For an EV or HEV designer, state-of-charge (SOC) estimation and energy recycle are significant issues. How to calculate the residual energy and estimate the rest of the journey are the key points they concerned. So, in this section, we introduce a new defined driving cycle (distance-based driving cycle, DBDC) to describe the relationship between vehicle speed and distance. The difference of the two definitions can be seen from Figure 1, in which no idle phase was presented in the given DBDC. (In Figure 1(a), the driving cycle was described by speed-time. In Figure 1(b), the driving cycle was described by speed-distance.)

Definition 1 (drive cycle, DC). DC is the set of data points representing vehicle speed versus time.

Definition 2 (distance-based drive cycle, DBDC). DBDC is the set of data points representing vehicle speed versus distance travelled from the start point in a given route.

\section{Generating DBDCs with Discrete Cosine Transform}

3.1. Splitting the Whole Route into Length-Fixed Segments. Nowadays, a lot of DC generation methodologies classify driving scenarios into several categories, for example, stopand-go, urban, suburban, rural, and highway. And then, they build DCs according to different scenarios [30-32]. However, the classification is intuitive and there is not a clear boundary or criterion existing for it. Particularly for electric special purpose vehicles, their driving mission profiles are very special. An adapted and representative driving cycle has to be built to facilitate the design of them. In fact, many factors, such as location, topography, driving characteristics, environment, and traffic, give their effects to the driving

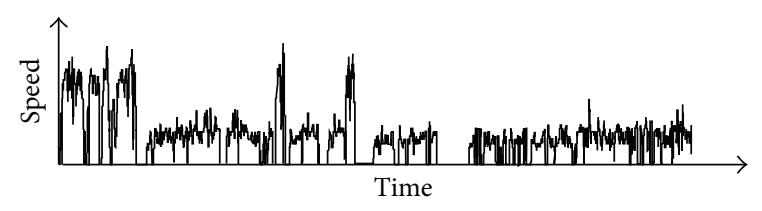

(a) Drive cycle

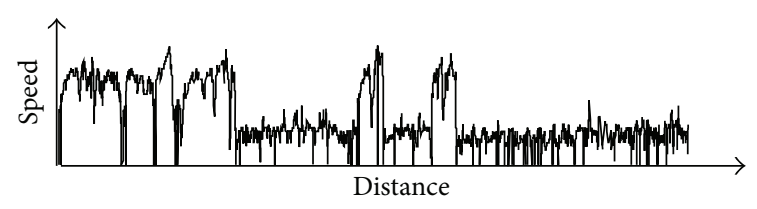

(b) Distance-based drive cycle

FIgURE 1: Drive cycle and distance-based drive cycle.

profiles. The complexities of the relationship between them make it impossible to construct an accurate driving profile with limited parameters. Researchers listed some important parameters and modeled the stochastic characteristics of a driving profile with these parameters. But the accuracy of them is not easy to be controlled and the parameters are difficult to be extended.

In this section, we separate the whole route into segments by distance. Each segment presents a length-fixed route, with which distance-based driving cycles can be freely constructed. For each segment, it is very easy to convert the representing manner of them from time domain to frequency domain. After changing, the number of the frequency component coefficients of the sequence is fixed (the lengths of the segments are fixed). So, we can easily calculate the probabilistic characteristics of it and rebuild a new driving cycle for EVs. As the frequency components are orthogonal, we can independently adjust the coefficients to facilitate the EV or HEV design.

3.2. Changing the Segments into Frequency Domain. Having divided the whole route into segments and expressed the segments in speed-distance manner, the discrete cosine transform can be used to change the segments into frequency components. Let $x(n)$ be the sequential data points representing vehicle speed versus distance, let $y(k)$ be the coefficients of different frequency components, let $N$ be the total number of the data points $x(n)$, and DBDCs of the route-segments can be changed into a series of components with different frequency coefficients:

$$
\begin{aligned}
& y(k)=w(k) \sum_{n=0}^{N-1} x(n) \cos \left(\frac{\pi(2 n+1) k}{2 N}\right) \\
& k=0,1, \ldots, N-1,
\end{aligned}
$$

where

$$
w(k)= \begin{cases}\frac{1}{\sqrt{N}} & k=0 \\ \sqrt{\frac{2}{N}} & 1 \leq k \leq N-1 .\end{cases}
$$




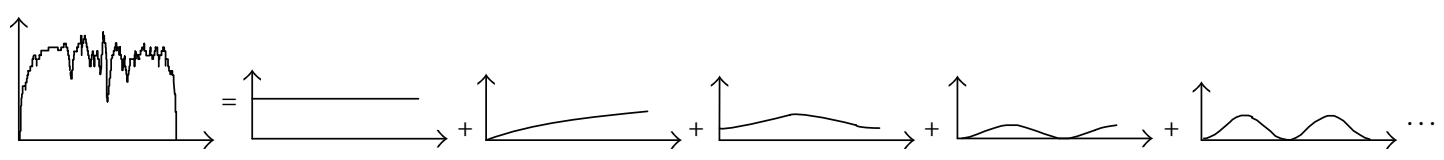

FIGURE 2: Discrete cosine transformation.

$y$ return the unitary discrete cosine transform of $x ; x$ and $y$ are the same size. The series is indexed from $n=0$ and $k=0$.

In fact, these frequency components are very useful for describing the internal and external parameters disturbing the constructed driving cycles. Generally speaking, the lowfrequency components are decided by terrain topology, traffic congestion. The medium-frequency components are decided by traffic flow, driving characteristics and the high-frequency components are decided by road condition, rapid driving maneuvers, spontaneous reactions, and so forth. We can see this in Figure 2.

3.3. Calculating Probability Functions. After changing the DBDC into frequency domain, a probability function (or probability density function) should be assigned to each coefficient of these frequency components, which will be used to generate random driving cycles. These probability functions are derived by interpolating data from driving profile analysis.

In this section, we implement one hundred times of tests with electric sanitation vehicles (ZLJ5071TSL), which belong to Beijing Sanitation Group Corporation. In the same work route, we recorded 100 groups of speed-distance data and divided them into length-fixed segments. After doing that, we changed these segments into frequency domain and calculated the distributions of the frequency component coefficients. For each of these coefficients, both of the largest value and smallest value of them were marked. We divided the interval of them into 10 parts and calculated the probability distributions for each of them. Having done this, we use the curve fitting function Cftool (Matlab) to create the probability surface to fit the inputs and outputs. The frequency of the coefficient values and the surface were shown in Figure 3, from which we can see that, with the increasing of the serial number of coefficient, the values and the ranges of it kept getting down. For example, the ranges of $y(0), y(10), y(50)$, and $y(100)$ are $360,315,90$, and 45 . This is decided by the energy-compaction property of DCT. So, we can present a route-segment with very few coefficients.

3.4. Reconstructing DBDC Sequence. Based on real statistical distributions of parameters, it is possible to generate a driving cycle that is statistically representative of real driving patterns. In this section, we use the inverse discrete cosine transform to reconstruct a sequence. The discrete cosine transform (DCT) coefficients were produced with the probability functions obtained in Section 3.3. Based on the frequency coefficients $y(k)$, we can calculate the segment sequence $x(n)$ and then reconstruct DBDC sequence by connecting different segments. The terminal sequence presents the driving cycle in a speed-distance manner.
Let $y(k)$ be the coefficients of different frequency components, let $x(n)$ be the sequential data points representing vehicle speed versus distance, and let $N$ be the total number of the data points $x(n)$; then, the DBDCs of the route-segments can be obtained by the formula below:

$$
\begin{array}{r}
x(n)=\sum_{k=0}^{N-1} w(k) y(k) \cos \left(\frac{\pi(2 n+1) k}{2 N}\right) \\
n=0,1, \ldots, N-1,
\end{array}
$$

where

$$
w(k)= \begin{cases}\frac{1}{\sqrt{N}} & k=0 \\ \sqrt{\frac{2}{N}} & 1 \leq k \leq N-1\end{cases}
$$

and $N=$ length $(x)$, which is the same as length $(y)$. The series is indexed from $n=0$ and $k=0$.

\section{Algorithm Analysis}

\subsection{Orthogonality Analysis}

Definition 3 (record interval, $M$ ). The record interval $M$ is a fixed distance between two successive speed records. In general, the distance $M$ is one meter.

Definition 4 (DBDC sequence, $x(n)$ ). The DBDC sequence $x(n), n \in[0, N-1]$, is a set of sequential speed-distance pairs. $n$ is the serial number; the value of $x(n)$ is the speed at the $n$th point. $x(n)$ can be used to represent the vehicle speed versus distance traveled from the start point in a given route.

Definition 5 (DBDC frequency coefficient sequence, $y(k)$ ). The DBDC frequency coefficient sequence $y(k), k \in[0, N-$ $1]$, is the set of frequency coefficients. $k$ is the serial number; $y(k)$ is the value of the $k$ th coefficient.

Theorem 6. Let $x(n), n \in[0, N-1]$, be a DBDC sequence. Let $y(k), k \in[0, N-1]$, be the coefficient sequence of $x(n)$. If the coefficient sequence $y(k)$ was obtained from sequence $x(n)$ by the discrete cosine transform DCT-II, then, the coefficients of $y(k)$ are orthogonal.

Proof. According to the definition of DCT-II,

$$
\begin{aligned}
& y(0)=\sqrt{\frac{1}{N}} \sum_{n=0}^{N-1} x(n), \\
& y(k)=\sqrt{\frac{2}{N}} \sum_{n=0}^{N-1} x(n) \cos \left(\frac{\pi(2 n+1) k}{2 N}\right) .
\end{aligned}
$$



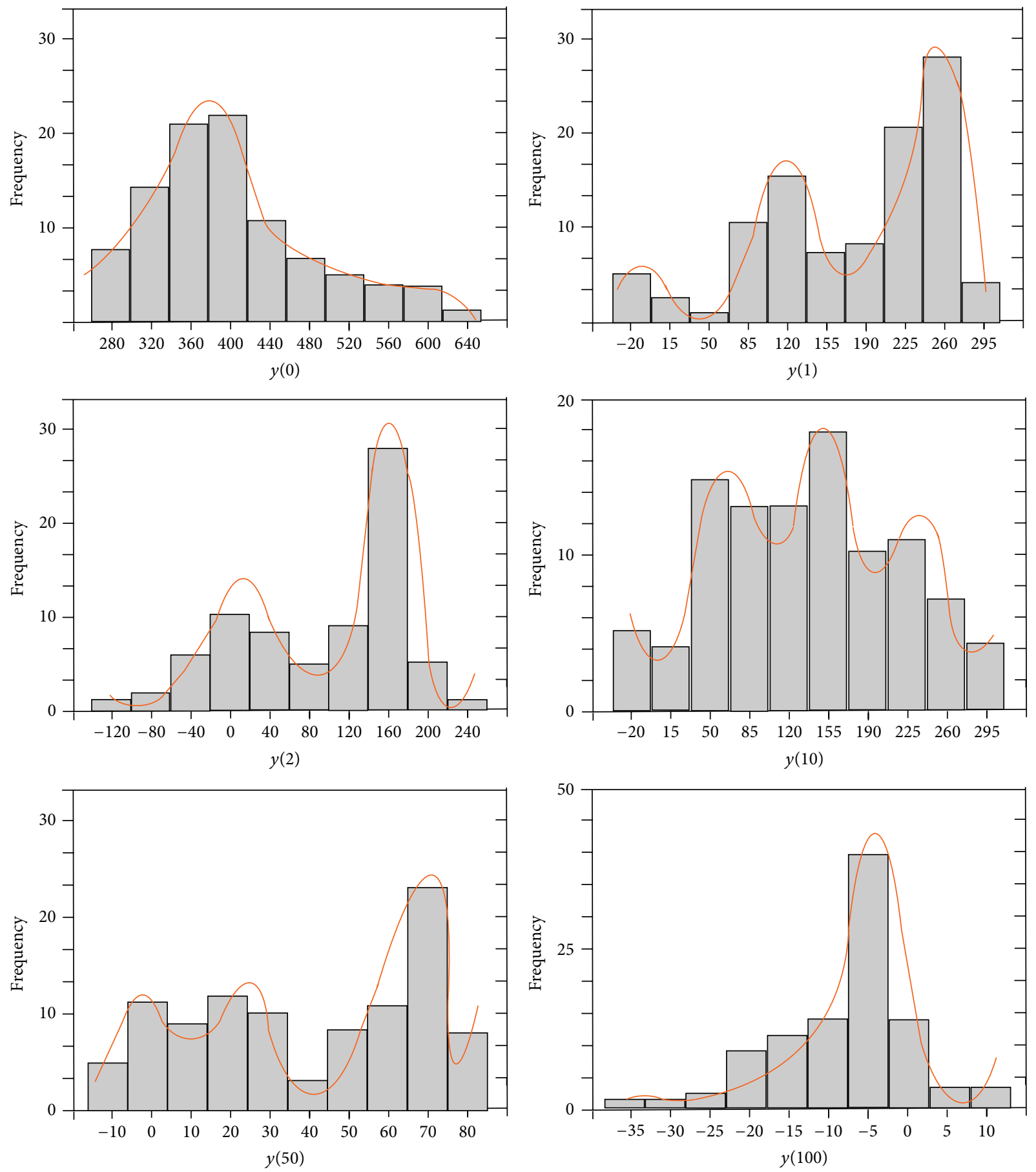

FIGURE 3: Statistical distributions of $y(0), y(1), y(2), y(10), y(50)$, and $y(100)$.

The base vector of them is $\{\sqrt{1 / N}, \sqrt{2 / N} \cos (\pi(2 n+$ 1) $k / 2 N)\}$.

According to the Chebyshev polynomials,

$$
\begin{aligned}
T_{0}(p) & =\sqrt{\frac{1}{N}}, \\
T_{k}\left(z_{n}\right) & =\sqrt{\frac{2}{N}} \cos \left[k \arccos \left(z_{n}\right)\right] .
\end{aligned}
$$

$T_{k}\left(z_{n}\right)$ is a polynomial represented by parameters $k$ and $z_{n}$. The $N$ th polynomial of it is

$$
T_{N}\left(z_{n}\right)=\sqrt{\frac{2}{N}} \cos \left[N \arccos \left(z_{n}\right)\right] .
$$

If $T_{N}\left(z_{n}\right)=0$ then

$$
z_{n}=\cos \frac{(2 n+1) \pi}{2 N} .
$$




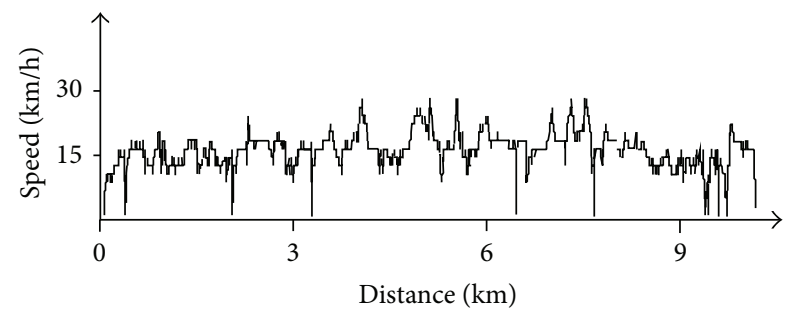

(a) Distance-based driving cycle

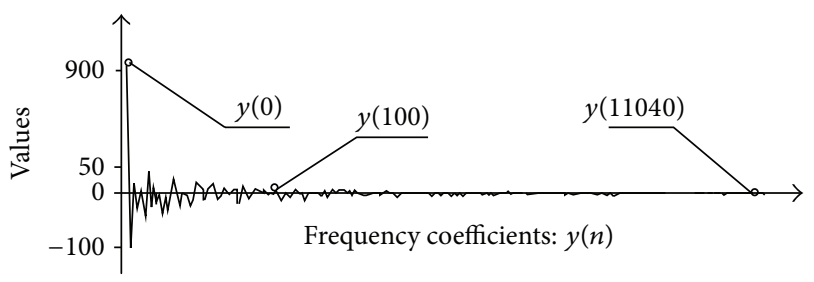

(b) The DCT coefficients

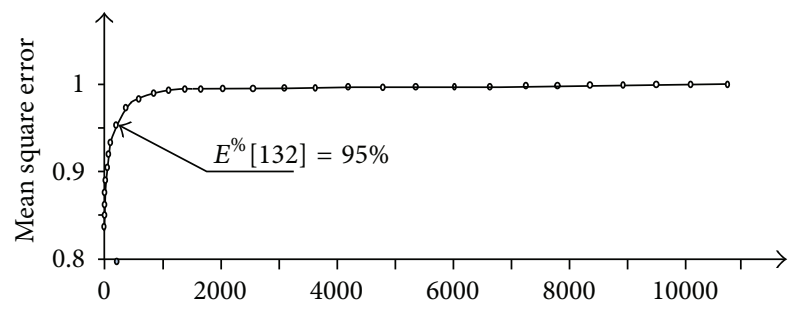

(c) The relative energy of $E^{\%}[c]$

FIGURE 4: The energy-compaction property of the DCT.

Bringing it in $T_{N}\left(z_{n}\right)$, we get

$$
\begin{aligned}
T_{k} & =\sqrt{\frac{2}{N}} \cos \left\{k \arccos \left[\cos \frac{(2 n+1) \pi}{2 N}\right]\right\} \\
& =\cos \frac{(2 n+1) k \pi}{2 N} .
\end{aligned}
$$

Obviously, it is the same with the base vector. Since the Chebyshev polynomial is orthogonal polynomial, the DBDC transformation is orthogonal too.

4.2. Energy Analysis. In fact, the key feature of DCT is the property of energy compaction, in which most of the information in a given signal can be concentrated in a small number of low-frequency components. DCT is closely related to the discrete Fourier transform (DFT), but by using only real numbers [23]. We can accurately reconstruct a sequence with only a small number of DCT coefficients. In this way, the DBDC sequence $(x(n), n \in[1, N])$ can be expressed by reduced frequency components $\left(y(k), k \in\left[1, K^{*}\right]\right)$, where $K^{*} \ll N$.

In Figure 4, we plot the speed-distance picture of an electric sanitation vehicle. Totally 11041 data points are obtained and each of them is a speed-distance pair. We did not split the route into segments and the whole route was changed from time domain into frequency domain. After transforming, the obtained coefficients sequence $y(n)$ was shown in Figure 4(b), from which we can see that most of
TABLE 1: Parameters of ZLJ5071TSL electric sanitation vehicle.

\begin{tabular}{lc}
\hline Vehicle type & ZLJ5071TSL sanitation EV \\
Chassis type & BJ1071VDE0A-1 \\
Max. mass & $7495 \mathrm{~kg}$ \\
Driving battery & $384 \mathrm{~V} / 200 \mathrm{Ah} \mathrm{Li}$-ion \\
Electromotor rated power & $60 / 110 \mathrm{~kW}$ \\
Fan rated power & $28 \mathrm{~kW} / 2900 \mathrm{rpm}$ \\
Max. velocity & $80 \mathrm{~km} / \mathrm{h}$ \\
Max. travel distance (running) & $150 \mathrm{~km}$ \\
Max. travel distance (working) & $50 \mathrm{~km}$ \\
Cleaning width & $3 \mathrm{~m}$ \\
Garbage bin & $5 \mathrm{~m}^{3}$ \\
Gradient coefficient $(\theta)$ & 0 \\
Drag coefficient $\left(c_{d}\right)$ & 0.33 \\
Tire rolling resistance $(\mu)$ & 0.015 \\
Front area $\left(A_{\text {front }}\right)$ & $2.5 \mathrm{~m}^{2}$ \\
Density of air $\left(\rho_{\text {air }}\right)$ & $1.2 \mathrm{~kg} / \mathrm{m}^{3}$ \\
\hline
\end{tabular}

the energy of the sequence is concentrated into the lowfrequency coefficients ahead.

For further indicating the energy-compaction property, we calculated the relative energies between the DBDC sequence $x(n)$ and the new obtained sequence $x_{c}(n)$ produced by cutting down the high-frequency components from $y(c+1)$ to $y(N-1)$. Let $E^{\%}[c]$ be the relative energy; then

$$
E^{\%}[c]=\frac{\sum_{n=0}^{c}\left|x_{c}(n)\right|}{\sum_{n=0}^{N-1}|x(n)|} .
$$

From Figure 4(c), we can see that 95\% energy of the DBDC sequence $x(n)$ can be expressed by $1.19 \%(132 / 11041)$ frequency components of sequence $y(n)$.

\section{Experimental Verification}

5.1. DBDC Generation. In this section, one hundred hours $(800 \mathrm{~km})$ of distance-based driving cycle data were acquired in the city Beijing in February and March of 2014. The electric sanitation vehicles used for test are ZLJ5071TSL, the equipment number of which is 400 . We obtained these data with twenty electric sanitation vehicles, the parameters of which were listed in Table 1.

The probability functions of 150 coefficients were extrapolated from the test data of ZLJ5071TSL using a VC++ script. The whole DBDC consists of several route-segments. Each of them was constructed with the method above. Figure 5 shows the generated DBDC snippets. For further investigating and comparing the recorded data and the created driving profile, we showed the speed-acceleration frequency distributions of the two DBDCs in Figure 6, in which the frequency coefficients were normalized. There are two frequency peaks ( ppeed $=35$, acceleration $=0 \mathrm{~m} / \mathrm{s}^{2}$; speed $=15$, acceleration $=0 \mathrm{~m} / \mathrm{s}^{2}$ ) which appeared in Figure 6(a). Similarly, two frequency peaks appeared in Figure 6(b) too. We can see that the frequency distributions of the two kinds of driving data are very similar. 


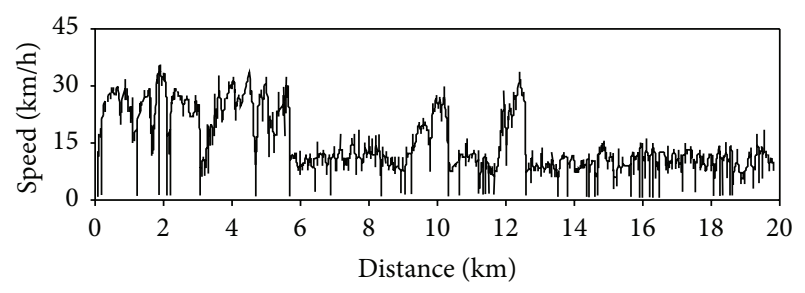

(a) Produced data

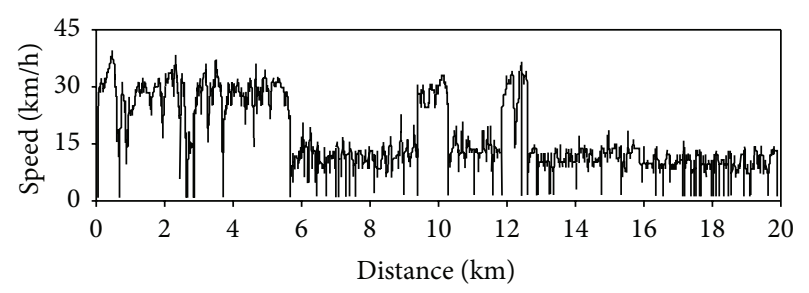

(b) Recorded data

FIGURE 5: The generated and recorded DBDC snippets.

5.2. Frequency Coefficient Analysis. In fact, one of the key superiorities of the proposed DBDC-construction method is the convenience on reconstructing the frequency and amplitude of the DBDC sequences. Having changed the driving cycle into frequency domain, we can reconstruct a new DBDC sequence easily by combining some of the coefficients which appeared in different DBDCs. For example, we showed original DBDC sequence $Y$ and some new sequences $(Y 1, Y 2, \ldots, Y 8)$ evolved from $Y$ in Figure 7 . Most of the new building sequences have only partly frequency components. For sequence $Y 1$, the sequence was constructed with lowfrequency components. It leads to a smooth profile and only structure information is left. For sequence $Y 7$, the sequence is constructed with high-frequency components. So, only details are left. In fact, the low-frequency components are mainly decided by terrain topology or traffic congestion and the high-frequency components are mainly decided by driving maneuvers or spontaneous reaction. Thus, we can freely examine the influences coming from terrain information or driving maneuvers by reconstructing the driving cycles with different coefficients.

5.3. Power Demand Analysis. We can further examine the physical impact of a driving profile by comparing the frequency features of the power demand. Firstly, we convert a DBDC into a duty cycle. Then, the internal and external forces are calculated with a vehicle dynamic model. Let $m$ be the vehicle mass, $a$ the acceleration, $g$ the gravitational acceleration, $\theta$ the road angle, $\mu$ the tire rolling resistance, $\rho_{\text {air }}$ the density of air, $v$ the velocity, $c_{d}$ the drag coefficient, $A_{\text {front }}$ the front area of the vehicle, $F_{\text {acc }}$ the external force for acceleration, $F_{\text {grav }}$ the projected normal force, $F_{\text {roll }}$ the rolling resistance, and $F_{\text {drag }}$ the aerodynamic drag force. Thus, we can present the dynamic model like this:

$$
\begin{aligned}
F_{\mathrm{acc}} & =m \times a, \\
F_{\mathrm{grav}} & =m \times g \times \sin \theta,
\end{aligned}
$$

TABLE 2: Parameters of the dynamic model.

\begin{tabular}{lc}
\hline Max. mass & $7495 \mathrm{~kg}$ \\
Gradient coefficient $(\theta)$ & 0 \\
Drag coefficient $\left(c_{d}\right)$ & 0.33 \\
Tire rolling resistance $(\mu)$ & 0.015 \\
Front area $\left(A_{\text {front }}\right)$ & $2.5 \mathrm{~m}^{2}$ \\
Density of air $\left(\rho_{\text {air }}\right)$ & $1.2 \mathrm{~kg} / \mathrm{m}^{3}$ \\
\hline
\end{tabular}

$$
\begin{gathered}
F_{\text {roll }}=\mu \times m \times g \times \cos \theta, \\
F_{\text {drag }}=\rho_{\text {air }} \times v^{2} \times c_{d} \times \frac{A_{\text {front }}}{2} .
\end{gathered}
$$

In Figure 8, we showed the frequency spectra of several changed driving profiles in power frequency distribution plot for the given electric sanitation vehicle, ZLJ5071TSL. The specifications of it were shown in Table 2. The driving profiles we used are sequences $Y, Y 1, Y 6$, and $Y 8$. We obtained them by adjusting the frequency coefficients of $Y$. The specific procedures and methods are shown in Figure 7.

From Figure 8 we can see that the changes in frequency or amplitude, for a given DBDC sequence, will lead to large changes on power distribution. And the changes are always useful for an electric vehicle designer. For example, in Figure $8(Y 1)$ and (Y6), if we leave only low-frequency components $(Y 1)$ or high-frequency components $(Y 6)$ of the given sequence $Y$, the range of the power demand will become very narrow. And the low-frequency components carry more power demand information. If we enlarge only partial components $(Y 8)$ and keep other components unchanged, the distribution of the power demand will change greatly. So, for an electric vehicle designer, it is a special point of view to analyze the effects of terrain topology, driving characteristics, road condition, and driving maneuvers by reconstructing DBDC with suitable coefficients.

5.4. Comparisons. For many traditional driving cycle developing methodologies, selecting test route, collecting real data, and constructing driving cycle are three major components. Generally speaking, the developed driving cycle provides a speed-time profile that is representative for specific driving characteristics. And the driving cycle is usually used for testing vehicles, determining the emission factors, and building a platform for government's emissions control testing. To clearly show the features of the proposed driving cycle developing method, we compare it with the traditional methods in three aspects: driving cycle expression manner, construction method, and error.

5.4.1. Comparison-1 (Driving Cycle Expression Manner). To compare the two different driving cycle expression manners (DC and DBDC), two separate trips of the same route are shown. In Figure 9, two different expression manners are exhibited. In (a), the driving cycle was displayed by distance and it provides the spatial correspondence. In (b), the driving cycle was illustrated in time and it showed the temporal 


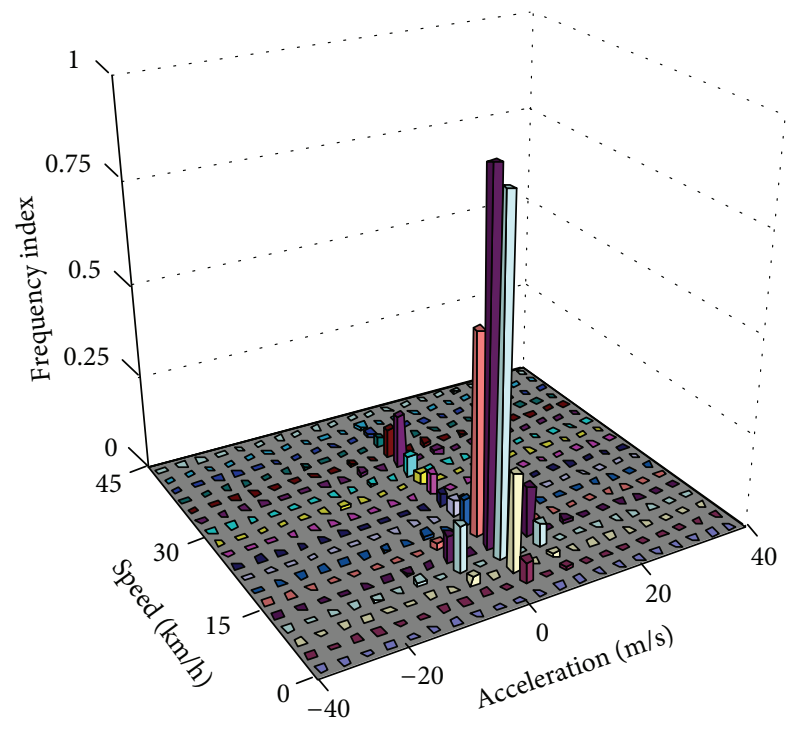

(a) Recorded data

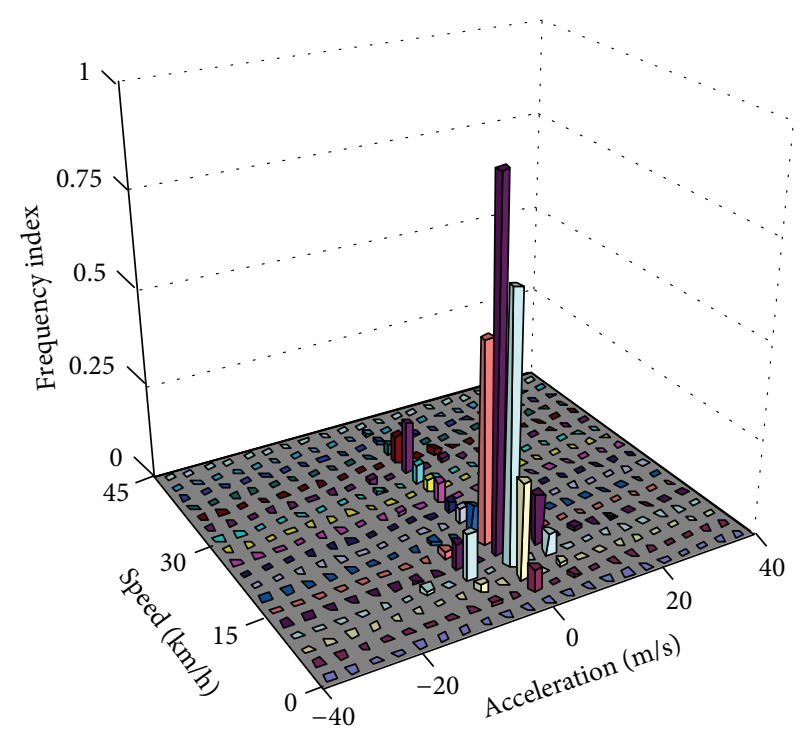

(b) Generated data

FIGURE 6: Speed-acceleration frequency distribution histograms for recorded data and generated data.

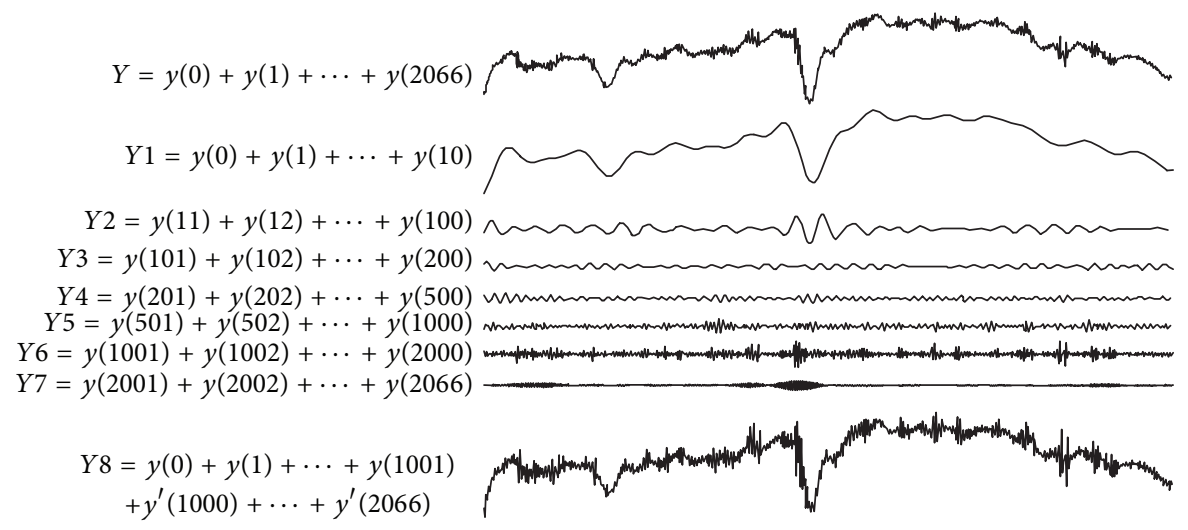

FIGURE 7: Different frequency coefficients.

correspondence. The trip of the upper driving cycle spanned for $3912 \mathrm{~s}$ and the one below spanned for 3624 s. If we change them into frequency domain, the low-frequency coefficients of them might be available for analyzing the global variation tendency. It contains the traffic signal location, grading, the time of the day, and so forth. From Figure 9(b), we can observe that the two driving cycles can not reflect the correlation of the trips (at the same time points, $600 \mathrm{~s}$ or $1750 \mathrm{~s}$, the speeds or accelerations have no correlation). However, in (a), the two driving cycles show better correspondence (at the same locations, $3.0 \mathrm{~km}$ or $9.9 \mathrm{~km}$, both of the two speed/accelerations decreased to zero). By comparing the distinct speed or accelerations at certain points which appeared in the same route, the distinct landmarks (e.g., the traffic lights and stop signs) and road conditions can be estimated. Thus, we can say that the distance-based representing method (used in this paper) is more suitable than traditional representing method to develop driving cycles for electric buses and special purpose vehicles, whose working or running routes are fixed.

5.4.2. Comparison-2 (Driving Cycle Construction Method). In fact, the most difficult task for developing a driving cycle is to consolidate the huge amount of driving data records into a representative driving cycle. In general, random selection approach is a commonly used method for constructing candidate driving cycles, with which many microtrips (bounded by idling periods) are randomly selected to form a driving cycle $[1,2,4-7]$. However, the random selection approach is only suitable for building DCs in a certain region or district. For electric buses and special purpose vehicles (such as electric sanitation vehicle, electric garbage truck, and electric tank car), the working and running routes of them are fixed and sequential. Constructing candidate driving cycles with the traditional random selection method will take the risk of losing most of the geography information of the routes, and 


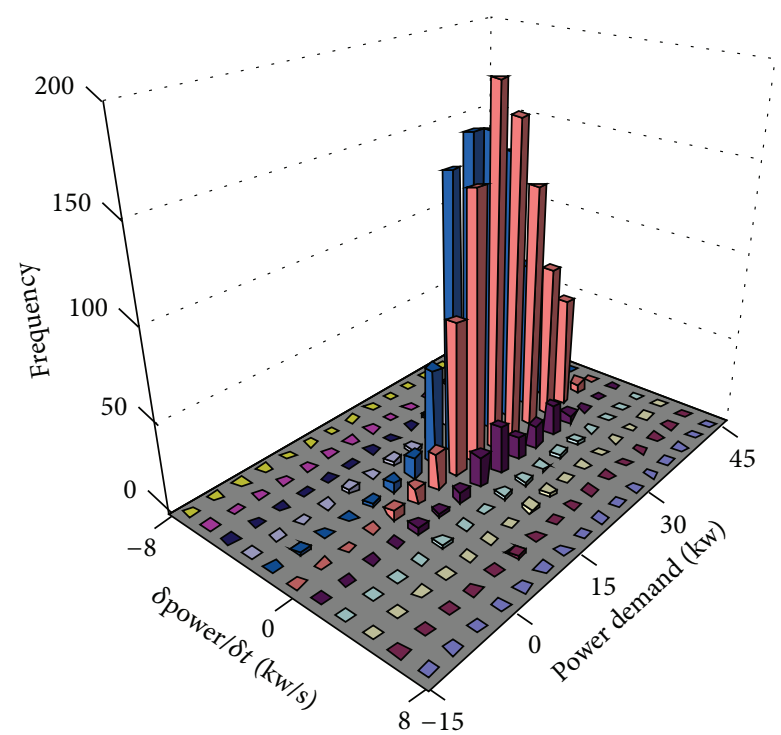

$(Y)$

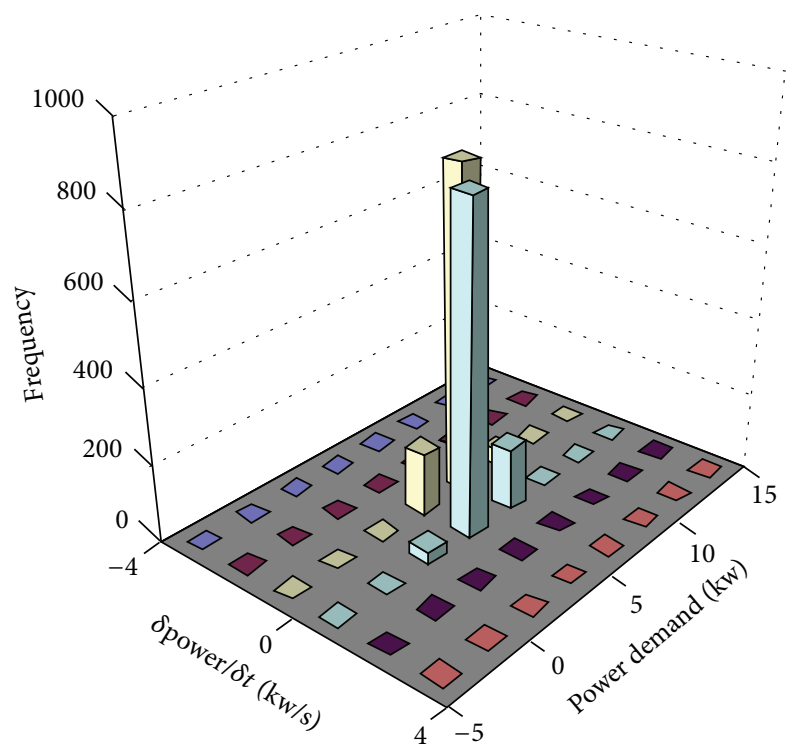

(Y6)

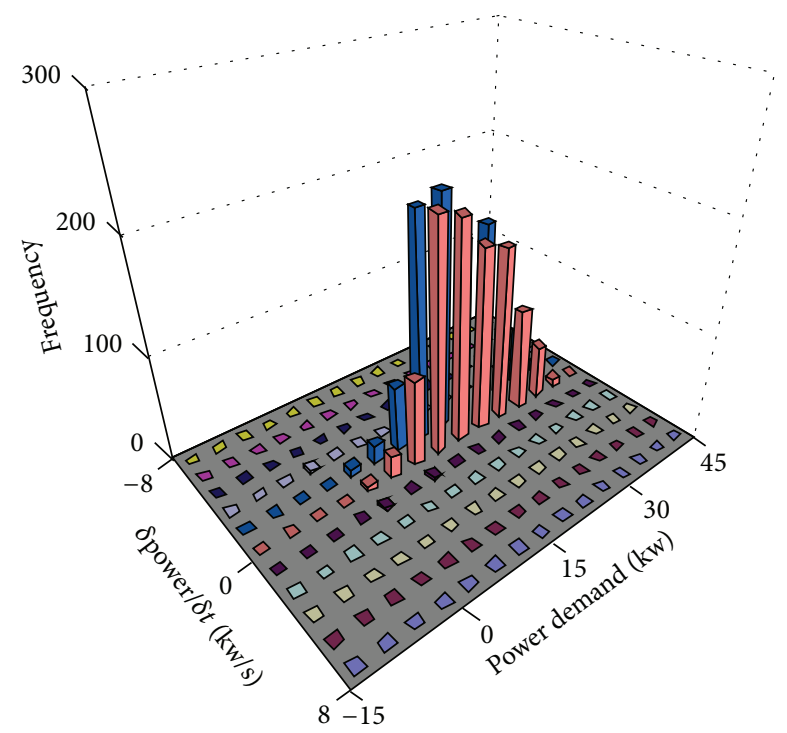

$(Y 1)$

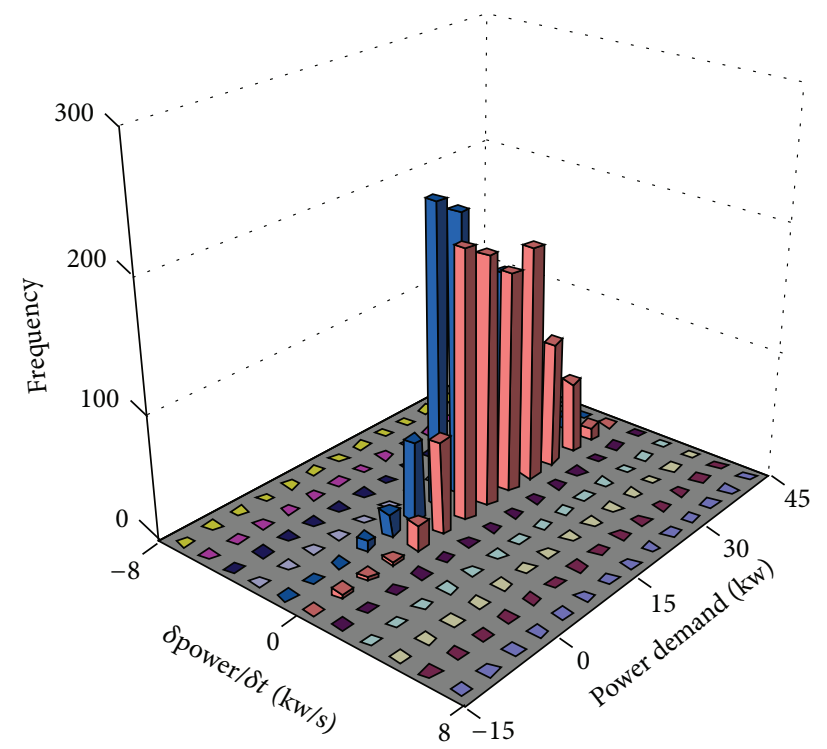

(Y8)

FIGURE 8: Frequency distribution of different power demands.

the obtained driving cycles can not reflect the sequential features of them. This can be seen in Figure 10, where three DBDCs were shown. In (a), the DBDC-1 was constructed with the random selection method. In (b), the DBDC-2 was constructed with the proposed method. In (c), the DBDC3 was obtained with the primary data by deleting the idling periods. The sequence of the microtrips reflects specific traffic-conditions that appeared in the route, so does DBDC2 , which was constructed with the proposed method. The difference of the two driving cycles, DBDC-3 and DBDC-2, is that DBDC-2 implied not only the sequence information of the microtrips but also the statistical information coming from all of the recorded data, while DBDC- 3 is just one time of running. However, in (a), since the random selection method is adopted, the order of the microtrips is randomly decided. Even though the values of the assessment parameters (appeared in Section 5.4.3) in the two driving cycles, DBDC-1 and DBDC-3, are very similar, the obtained driving cycle can not be used for evaluating the special EVs. Why?

The reason lies in the characteristics of EVs. We can see this from Figure 11, where a driving cycle and the associated duty cycle are shown. In the figure, the rotate speed RPM is used to illustrate the transient and dynamic performances involved in the power train system. The battery pack information, including the capacity, state-of-charge, and energy profiles, is also illustrated. From it we can find that, during the running process, the SOC or residual capacity of the battery pack keeps decreasing. For each of the microtrips, which appeared in the DBDC sequence, the corresponding SOC or residual capacity is different. If a demand of peak 

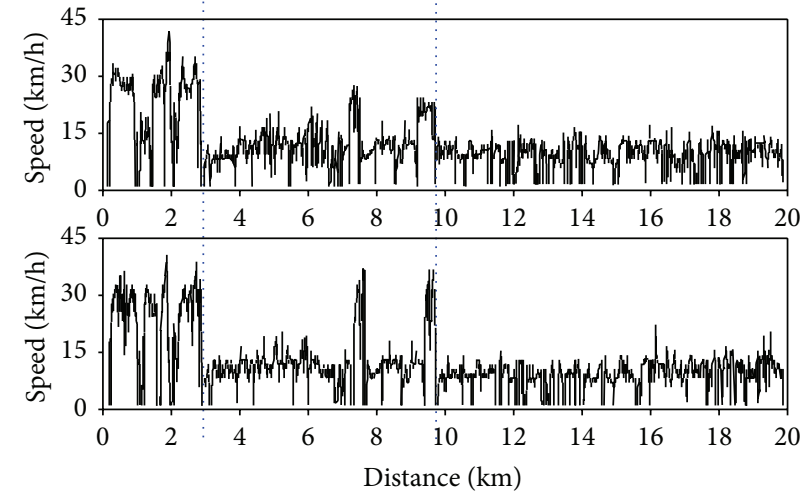

(a)
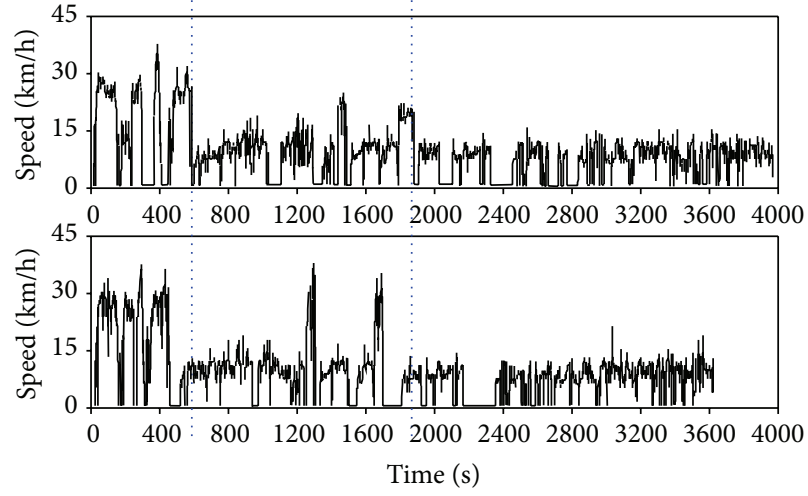

(b)

FIgURE 9: The different expression manners. (a) Plots are illustrated in distance. (b) Plots are illustrated in time.

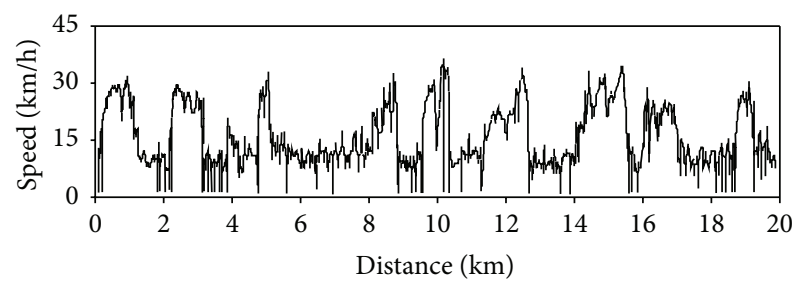

(a) Driving cycle DBDC-1 (constructed with the random selection method)

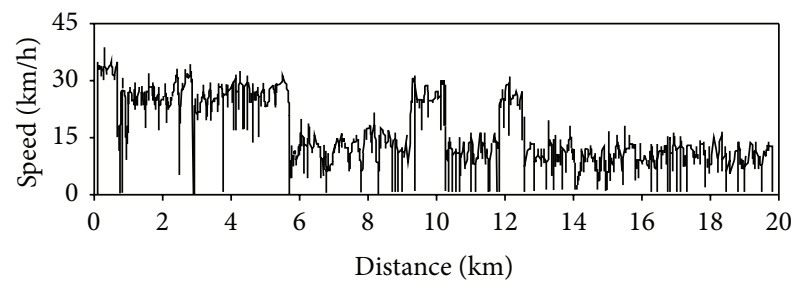

(b) Driving cycle DBDC-2 (constructed with the proposed method)

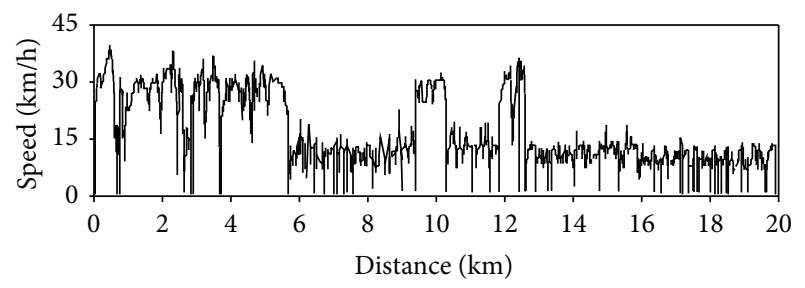

(c) Driving cycle DBDC-3 (constructed with the primary records)

Figure 10: The generated DBDCs (with different driving cycle construction methods).

power appeared in a microtrip close to the start point, where the residual capacity of the pack is sufficient, the demand will be fulfilled. However, if it appears in a microtrip close to the end, where no enough power can be supplied, the demand will not be fulfilled. Thus, the sequence of microtrips is a key factor for electric vehicles, whose working routes are fixed and sequential. It should not be randomly constructed.

Additionally, the energy-recovery characteristic of EV is another reason for us to discard the traditional random selection method. For an EV, the effectiveness of energyrecovery system changes with the battery pack status. At different positions (in a route), the residual capacity of the battery pack is different. Ignoring the sequence of microtrips and estimating SOC or residual capacity for an EV with energy-recovery system will lead to large errors. For example, if the battery pack is fully charged at the start point, a long distance of downhill will give no help for charging.

5.4.3. Comparison-3 (Errors). For most of the driving-cycle generation methods, after establishing a candidate cycle, the assessment parameters will be calculated and compared with the target statistics (the assessment criteria and parameters are derived and determined in the period of data collection). If each of the assessment parameters is different from the target mean values by less than a given value, $\epsilon$, the candidate cycle will be accepted. Otherwise, the next candidate cycle will be sampled and this exercise will repeat. In Table 3, we compare the errors of the five candidate driving cycles (constructed with the traditional method) with driving cycle, DBDC-2 (constructed with the proposed method). The assessment parameters employed in the comparison process are as follows.

(1) Average running speed (V1).

(2) Average acceleration of all acceleration phases $(a)$.

(3) Average deceleration of all deceleration phases $(d)$.

(4) Distance proportions of driving modes-acceleration $(\mathrm{Pa})$.

(5) Distance proportions of driving modes-deceleration (Pd).

(6) Average number of acceleration-deceleration changes $(N)$.

(7) Root mean square acceleration (RMS).

(8) Positive acceleration kinetic energy-PKE $\left(\mathrm{m} / \mathrm{s}^{2}\right)$.

By comparing the values of the eight parameters, we can see that the error of DBDC-2 is less than that of the 

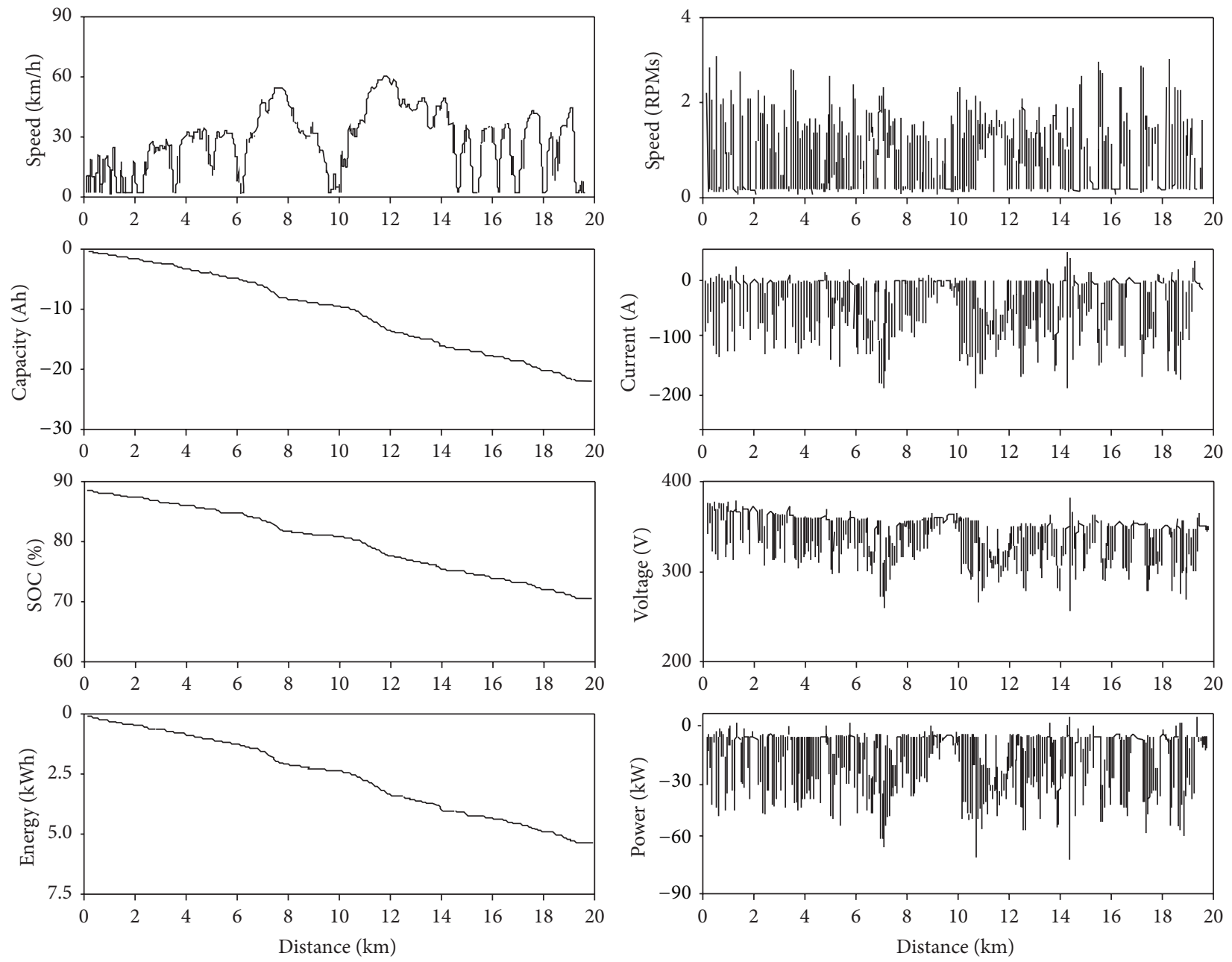

FIGURE 11: An example DBDC and the associated parameters.

TABLE 3: Assessment criteria and the values of them in candidate driving cycles and DBDC-2.

\begin{tabular}{|c|c|c|c|c|c|c|c|c|}
\hline $\mathrm{DC}$ & $V 1(\mathrm{~m} / \mathrm{s})$ & $a\left(\mathrm{~m} / \mathrm{s}^{2}\right)$ & $d\left(\mathrm{~m} / \mathrm{s}^{2}\right)$ & $\mathrm{Pa}(\%)$ & $\mathrm{Pd}(\%)$ & $N$ (time) & $\operatorname{RMS}\left(\mathrm{m} / \mathrm{s}^{2}\right)$ & $\operatorname{PKE}\left(\mathrm{m} / \mathrm{s}^{2}\right)$ \\
\hline Average & 5.71 & 0.45 & 0.48 & 38.62 & 35.66 & 125 & 0.54 & 0.16 \\
\hline Candidate-1 & 5.92 & 0.44 & 0.49 & 40.51 & 33.42 & 121 & 0.53 & 0.15 \\
\hline Candidate-2 & 5.45 & 0.45 & 0.49 & 39.65 & 35.06 & 114 & 0.56 & 0.17 \\
\hline Candidate-3 & 5.61 & 0.43 & 0.47 & 39.61 & 33.82 & 129 & 0.52 & 0.15 \\
\hline Candidate-4 & 5.97 & 0.44 & 0.48 & 39.72 & 34.12 & 119 & 0.54 & 0.16 \\
\hline Candidate-5 & 5.55 & 0.45 & 0.47 & 38.21 & 36.42 & 132 & 0.57 & 0.17 \\
\hline DBDC-2 & 5.72 & 0.45 & 0.48 & 38.88 & 35.61 & 127 & 0.55 & 0.16 \\
\hline
\end{tabular}

five candidate driving cycles. What is the reason? After all, both the average values of the eight parameters and the five candidate driving cycles are obtained with the recorded data in time domain, while DBDC-2 is constructed in frequency domain. The reason lies in the two different driving cycle construction methods. For the traditional construction method, the first step is to calculate the assessment measures for the collected data. And then, a candidate cycle will be developed by randomly selecting the microtrips from the microtrip pool. Finally, if the error between the candidate cycle and the assessment measure is very small, the candidate cycle will be reserved. Otherwise, a new construction process will begin. Since each of the microtrips can only be selected as a whole section, the probability for constructing a driving cycle whose test statistics are very close to the target statistics is very small.

On the contrary, for the proposed cycle construction method, the frequency coefficients of the route-segments are firstly calculated. And then, the probability functions of these coefficients are used to generate a random driving cycle. Since the driving cycle is directly constructed with the obtained 
probability functions and, at the same time, the probability functions are also the evaluation criterions in frequency domain, thus, the error between the assessment criteria with the obtained driving cycle will be very small. In fact, the error calculated in time domain and frequency domain is consistent. Thus, the test statistics of DBDC-2 are more close to the target statistics than other five candidate driving cycles.

\section{Conclusion}

For EVs, the major purpose for building driving cycle or duty cycle is to develop a method for assessing, predicting, and testing the performance and matching degree of batteries and vehicles. The road condition and driving habit take important roles in constructing a complex system to characterize or predict the long/short term effects of battery performance and life. In fact, many pieces of battery pack information, including capacity, state-of-charge, current, voltage, and state-of-health, have intensively relationships with frequency characteristics of DCs. For example, peak power and the frequency of duty pulses are primary factors affecting battery performance and life; the effectiveness of the energy-recovery system has great correlations on the high-frequency components of DCs. So, developing a novel DC expressing and constructing method to facilitate EV design is important for us.

\section{Conflict of Interests}

The authors declare that there is no conflict of interests regarding the publication of this paper.

\section{Acknowledgments}

The authors are grateful to the anonymous referees for their invaluable suggestions to improve the paper. This work was supported by National Science-Technology Support Plan Projects of China (2014BAG02B02), International S\&T Cooperation Program of China (ISTCP: 2015DFG81930), National Natural Science Foundation of China (51175043; 51205022), and China Postdoctoral Science Foundation funded project (2013M530534).

\section{References}

[1] R. Kumar, B. K. Durai, W. Saleh, and C. Boswell, "Comparison and evaluation of emissions for different driving cycles of motorcycles: a note," Transportation Research Part D: Transport and Environment, vol. 16, no. 1, pp. 61-64, 2011.

[2] S. Stockar, V. Marano, M. Canova, G. Rizzoni, and L. Guzzella, "Energy-optimal control of plug-in hybrid electric vehicles for real-world driving cycles," IEEE Transactions on Vehicular Technology, vol. 60, no. 7, pp. 2949-2962, 2011.

[3] T. Austin, F. DiGenova, T. Carlson, R. Joy, K. Gianolini, and J. Lee, "Characterization of driving patterns and emissions from light-duty vehicles in California," Tech. Rep., Sierra Research Inc., Sacramento, Calif, USA, 1993.

[4] J. Lin and D. A. Niemeier, "Estimating regional air quality vehicle emission inventories: constructing robust driving cycles," Transportation Science, vol. 37, no. 3, pp. 330-346, 2003.
[5] M. Kuhler and D. Karstens, "Improved driving cycle for testing automotive exhaust emissions," SAE Technical Paper 780650, 1978.

[6] J. Wu, C.-H. Zhang, and N.-X. Cui, "Fuzzy energy management strategy for a hybrid electric vehicle based on driving cycle recognition," International Journal of Automotive Technology, vol. 13, no. 7, pp. 1159-1167, 2012.

[7] S. H. Kamble, T. V. Mathew, and G. K. Sharma, "Development of real-world driving cycle: case study of Pune, India," Transportation Research Part D: Transport and Environment, vol. 14, no. 2, pp. 132-140, 2009.

[8] D. Jung, J. Bang, S. Choi, H. Choi, and K. Min, “Optimization algorithm for diesel engine operating parameters based on a vehicle driving test cycle," Journal of Mechanical Science and Technology, vol. 27, no. 7, pp. 2171-2179, 2013.

[9] J.-S. Won and R. Langari, "Fuzzy torque distribution control for a parallel hybrid vehicle," Expert Systems, vol. 19, no. 1, pp. 4-10, 2002.

[10] C.-C. Lin, H. Peng, J. W. Grizzle, and J.-M. Kang, "Power management strategy for a parallel hybrid electric truck," IEEE Transactions on Control Systems Technology, vol. 11, no. 6, pp. 839-849, 2003.

[11] P. Rodatz, G. Paganelli, A. Sciarretta, and L. Guzzella, "Optimal power management of an experimental fuel cell/supercapacitor-powered hybrid vehicle," Control Engineering Practice, vol. 13, no. 1, pp. 41-53, 2005.

[12] S. Delprat, T. Guerra, and J. Rimaux, "Optimal control of a parallel powertrain: from global optimization to real time control strategy," in Proceedings of the IEEE 55th Vehicular Technology Conference (VTC '02), vol. 4, pp. 2082-2088, 2002.

[13] M.-J. Kim and H. Peng, "Power management and design optimization of fuel cell/battery hybrid vehicles," Journal of Power Sources, vol. 165, no. 2, pp. 819-832, 2007.

[14] D. Assanis, G. Delagrammatikas, R. Fellini et al., "An optimization approach to hybrid electric propulsion system design," Mechanics of Structures and Machines, vol. 27, no. 4, pp. 393-421, 1999.

[15] W. Gao and S. K. Porandla, "Design optimization of a parallel hybrid electric powertrain," in Proceedings of the IEEE Vehicle Power and Propulsion Conference (VPPC '05), pp. 530-535, September 2005.

[16] B. Zhang, Z. Chen, C. Mi, and Y. L. Murphey, "Multi-objective parameter optimization of a series hybrid electric vehicle using evolutionary algorithms," in Proceedings of the 5th IEEE Vehicle Power and Propulsion Conference (VPPC '09), pp. 921-925, September 2009.

[17] X. Liu, Y. Wu, and J. Duan, "Optimal sizing of a series hybrid electric vehicle using a hybrid genetic algorithm," in Proceedings of the IEEE International Conference on Automation and Logistics, pp. 1125-1129, Jinan, China, August 2007.

[18] S. Campanari, G. Manzolini, and F. Garcia de la Iglesia, "Energy analysis of electric vehicles using batteries or fuel cells through well-to-wheel driving cycle simulations," Journal of Power Sources, vol. 186, no. 2, pp. 464-477, 2009.

[19] J. Lin and D. A. Niemeier, "Regional driving characteristics, regional driving cycles," Transportation Research Part D: Transport and Environment, vol. 8, no. 5, pp. 361-381, 2003.

[20] S. Shahidinejad, E. Bibeau, and S. Filizadeh, "Statistical development of a duty cycle for plug-in vehicles in a North American urban setting using fleet information," IEEE Transactions on Vehicular Technology, vol. 59, no. 8, pp. 3710-3719, 2010. 
[21] A. Sciarretta and L. Guzzella, "Control of hybrid electric vehicles," IEEE Control Systems Magazine, vol. 27, no. 2, pp. 60-70, 2007.

[22] M. Fugel, T. Kassel, and F. Kucukay, "Simulation eines parallelen hybridantriebs im kundeneinsatz," in VDI-Berichte, vol. 2030, pp. 125-149, 2008.

[23] N. Ahmed, T. Natarajan, and K. R. Rao, "Discrete cosine transform," IEEE Transactions on Computers, vol. 23, no. 1, pp. 90-93, 1974.

[24] A. Ravey, N. Watrin, B. Blunier, D. Bouquain, and A. Miraoui, "Energy-source-sizing methodology for hybrid fuel cell vehicles based on statistical description of driving cycles," IEEE Transactions on Vehicular Technology, vol. 60, no. 9, pp. 4164-4174, 2011.

[25] B. Y. Liaw and M. Dubarry, "From driving cycle analysis to understanding battery performance in real-life electric hybrid vehicle operation," Journal of Power Sources, vol. 174, no. 1, pp. 76-88, 2007.

[26] K. Brundell-Freij and E. Ericsson, "Influence of street characteristics, driver category and car performance on urban driving patterns," Transportation Research Part D: Transport and Environment, vol. 10, no. 3, pp. 213-229, 2005.

[27] J.-H. Tsai, H.-L. Chiang, Y.-C. Hsu, B.-J. Peng, and R.-F. Hung, "Development of a local real world driving cycle for motorcycles for emission factor measurements," Atmospheric Environment, vol. 39, no. 35, pp. 6631-6641, 2005.

[28] B. H. Wang, Y. G. Luo, and J. W. Zhang, "Simulation of city bus performance based on actual urban driving cycle in China," International Journal of Automotive Technology, vol. 9, no. 4, pp. 501-507, 2008.

[29] V. Schwarzer and R. Ghorbani, "New opportunities for largescale design optimization of electric vehicles using GPU technology," in Proceedings of the 7th IEEE Vehicle Power and Propulsion Conference (VPPC '11), pp. 1-6, September 2011.

[30] P. H. Nguyen, E. Hoang, and M. Gabsi, "Performance synthesis of permanent-magnet synchronous machines during the driving cycle of a hybrid electric vehicle," IEEE Transactions on Vehicular Technology, vol. 60, no. 5, pp. 1991-1998, 2011.

[31] T.-K. Lee, B. Adornato, and Z. S. Filipi, "Synthesis of realworld driving cycles and their use for estimating PHEV energy consumption and charging opportunities: case study for midwest/U.S," IEEE Transactions on Vehicular Technology, vol. 60, no. 9, pp. 4153-4163, 2011.

[32] E. Tazelaar, J. Bruinsma, B. Veenhuizen, and P. van den Bosch, "Driving cycle characterization and generation, for design and control of fuel cell buses," World Electric Vehicle Journal, vol. 3, pp. 1-8, 2009. 


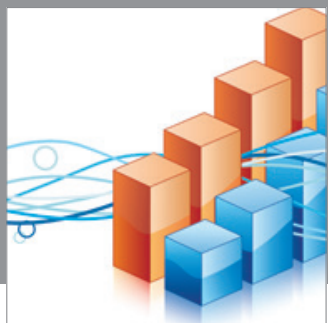

Advances in

Operations Research

mansans

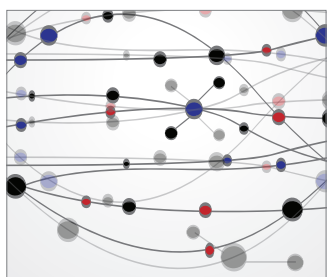

The Scientific World Journal
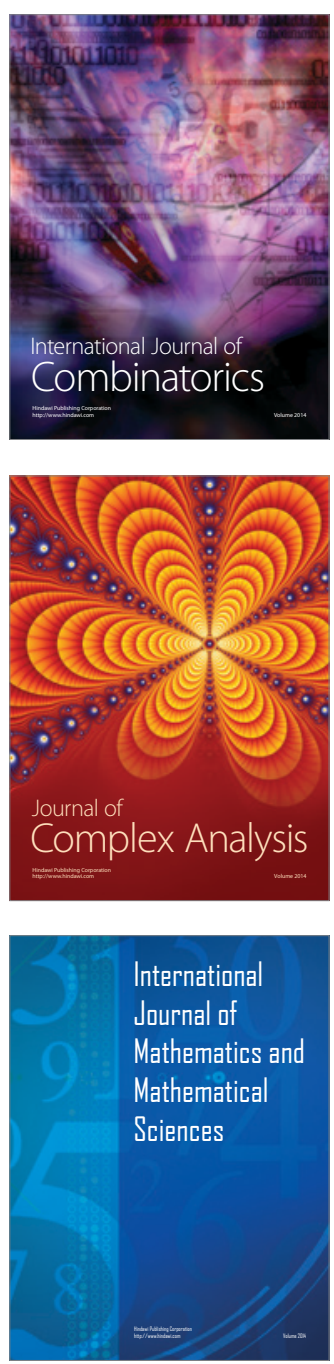
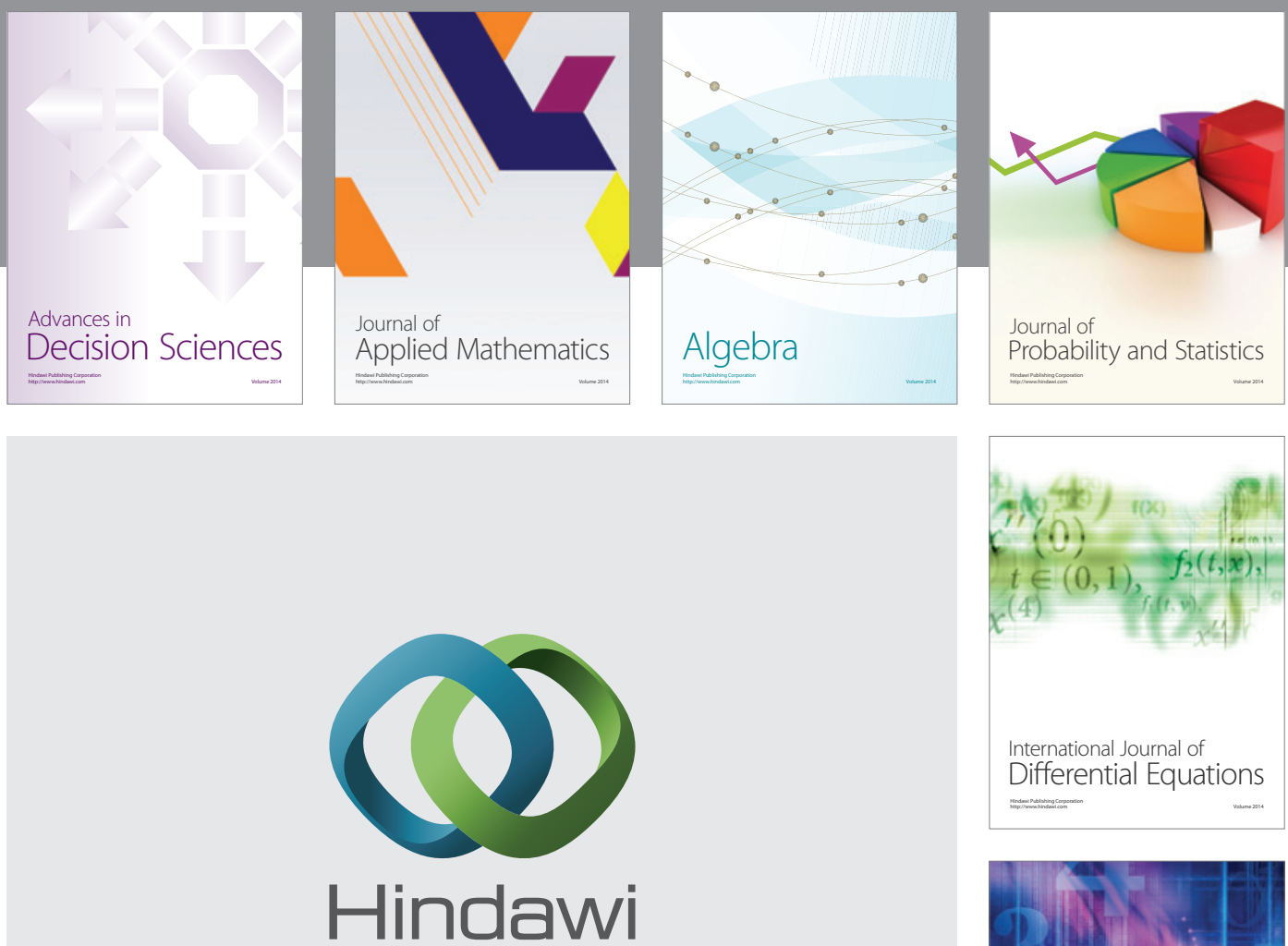

Submit your manuscripts at http://www.hindawi.com
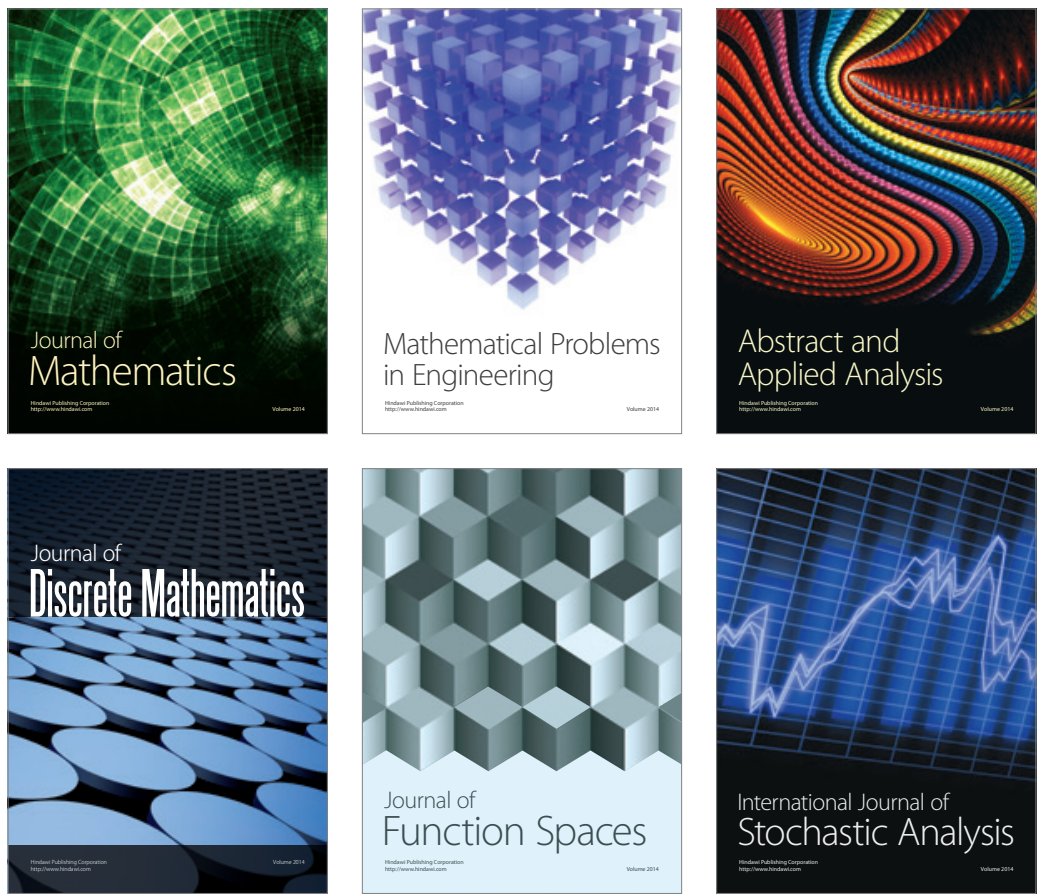

Journal of

Function Spaces

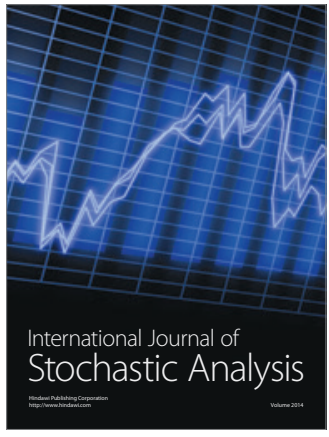

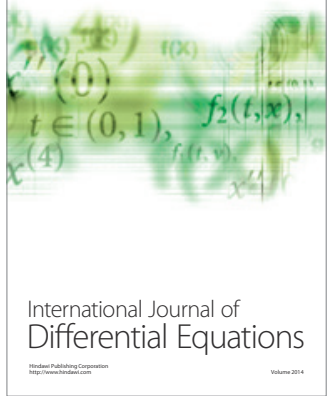
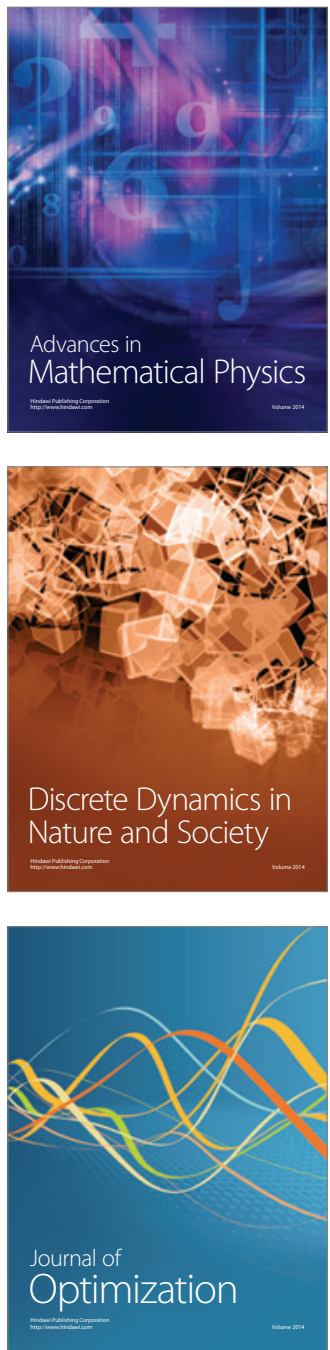